\title{
Borboletas (Lepidoptera, Papilionoidea e Hesperioidea) do Parque Estadual do Espinilho e entorno, Rio Grande do Sul, Brasil ${ }^{1}$
}

\author{
Maria O. Marchiori ${ }^{2} \&$ Helena P. Romanowski ${ }^{2}$
}

\begin{abstract}
${ }^{1}$ Contribuição número 500 do Programa de Pós-Graduação em Biologia Animal, Instituto de Biociências, Universidade Federal do Rio Grande do Sul.

2 Laboratório de Ecologia de Insetos, Departamento de Zoologia, Instituto de Biociências, Universidade Federal do Rio Grande do Sul. Avenida Bento Gonçalves 9500, Prédio 43435, 91501-970 Porto Alegre, Rio Grande do Sul, Brasil.

E-mail: mariosti2@hotmail.com; hpromano@ufrgs.br
\end{abstract}

\begin{abstract}
Butterflies (Lepidoptera, Papilionoidea e Hesperioidea) from Espinilho State Park and surroundings, Rio Grande do Sul, Brazil. This paper provides the first species list of butterflies from Espinilho

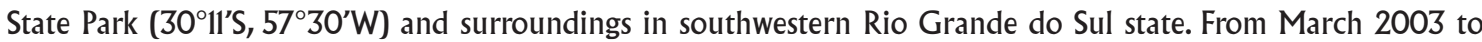
January 2004, four field expeditions were carried out, lasting five days each. The study covered areas with "Savana Estépica Parque" vegetation and riparian forest inside the park boundary and on its surroundings. The list included 97 species, 10 of which are new records for Rio Grande do Sul.

KEY WORDS. Lycaenidae; Nymphalidae; protected areas; riparian forest; Savana Estépica Parque.
\end{abstract}

RESUMO. O presente trabalho apresenta uma relação das espécies de borboletas que ocorrem no Parque Estadual do Espinilho $\left(30^{\circ} 11^{\prime} S, 7^{\circ} 30^{\prime} \mathrm{W}\right.$ ) e entorno no extremo sudoeste do Rio Grande do Sul. Foram realizadas quatro expedições a campo, com duração de cinco dias cada, entre março de 2003 e janeiro de 2004. As amostragens contemplaram áreas com vegetação de Savana Estépica Parque e Mata Ciliar dentro dos limites do parque e entorno. Foi elaborada uma listagem com 97 espécies de borboletas, havendo 10 novos registros para o Rio Grande do Sul.

PALAVRAS-CHAVE. Lycaenidae; Mata Ciliar; Nymphalidae; Savana Estépica Parque; unidades de conservação.

A diversidade biológica vem sendo perdida em ritmo ace lerado (WILSON 1997). Soma-se a isto o pouco conhecimento que temos sobre nossos ecossistemas nativos, o que limita as discussões e o desenvolvimento de planos de conservação dos recursos naturais existentes (MorelLato 1992). As Unidades de Conservação (UCs), notadamente as de proteção integral, são um componente essencial para a conservação in situ da biodiversidade(SOULÉ \& TERBORGH 1999). Estudos recentes (BRUNER et al. 2001) sugerem que mesmo as UCs deficientes em implantação e manejo são mais efetivas na conservação do que áreas não protegidas, podendo ser consideradas locais privilegiados para a realização de pesquisas científicas (BRITo et al. 1999).

O Parque Estadual do Espinilho (PEE), localizado no município de Barra do Quarai, extremo sudoeste do Rio Grande do Sul, abriga uma formação vegetal de alta singularidade ecológica: a Savana Estépica Parque (Veloso et al. 1991). Esta vegetação é homóloga a que ocupa as províncias de Corrientes e Entre Rios, na Argentina (RAmBo 1994). Ocorre no Paraguai e no norte do Chile, porém no Brasil, existe somente no município de Barra do Quarai. O parque também abriga formações de
Floresta Estacional Decidual Aluvial, aqui doravante chamadas de Matas Ciliares. Estas constituem um sistema de grande diversidade biológica (BRown JR. 2001), servindo de corredores e refúgios para muitas espécies. Apesar disto, poucos estudos tem sido realizados neste ambiente, sobretudo no Rio Grande do Sul (BUDKe et al. 2004).

O PEE, apesar de ser considerado uma área prioritária à conservação, pela fragilidade e importância dos ecossistemas que abriga, encontra-se insularizado devido às intensas atividades de agricultura e pecuária, características da região onde se situa. Contudo, seu entorno apresenta, nas margens dos rios Uruguai e Quarai, Matas Ciliares relativamente preservadas e de grande porte (VELoso et al. 1991), além de al gumas pequenas áreas disjuntas de Savana Estépica Parque localizadas dentro de propriedades particulares. O conhecimento da fauna que ocorre nestas áreas de entorno torna-se importante dada à reduzida área do PEE e como um possível referencial para seu próprio monitoramento.

O objetivo do presente estudo foi realizar um inventário das espécies de borboletas que ocorrem no PEE e entorno. In- 
ventário este de caráter inédito tanto para a UC como para a região de Barra do Quarai. Pretende-se, com isto, contribuir não somente para o conhecimento da fauna que ocorre no parque, como também para ampliação do conhecimento sobre a lepidopterofauna do Rio Grande do Sul.

\section{MATERIAL E MÉTODOS}

\section{Área de Estudo}

O Parque Estadual do Espinilho ( $30^{\circ} 11^{\prime} \mathrm{S}, 57^{\circ} 30^{\prime} \mathrm{W}$ ), com uma área de 1.617,14 ha, localiza-se no município de Barra do Quarai, extremo sudoeste do Rio Grande do Sul. Inserido na área de influência da microbacia do arroio Quarai-chico, às margens do rio Uruguai, o parque dista $6 \mathrm{~km}$ de Barra do Quarai e $765 \mathrm{~km}$ de Porto Alegre, capital do Estado.

O clima da região é do tipo Cfa de Köppen, subtropical úmido, sem estiagem, com médias anuais de precipitação de 1300 mm e temperatura de $23,4^{\circ} \mathrm{C}$ (МотА 1951), porém as temperaturas extremas podem ser negativas no inverno e passar de $35^{\circ} \mathrm{C}$ no verão. Os valores mensais de umidade variam de 60 a $70 \%$, indicando a existência, geralmente durante o verão, de um período seco que as normais de chuva não registram (SAnTos 1991).

A área do parque apresenta dois tipos de fitofisionomia: Savana Estépica Parque (SE) e Mata Ciliar (MC). A Savana Estépica Parque é composta por árvores afastadas umas das outras de forma homogênea, não havendo sobreposição de copas e permitindo o desenvolvimento de um tapete gramíneolenhoso (Veloso et al. 1991). É formada principalmente por uma associação de três leguminosas arbóreas, espinhosas e caducifólias que raramente superam $5 \mathrm{~m}$ de altura: Prosopis affinis Spreng., Prosopis nigra (Griseb.) Hieron. e Acacia caven (Mol.) Mol. (MARCHIORI et al. 1985). A Mata Ciliar compõe-se principalmente por representantes das famílias Myrtaceae e Fabaceae. Distribui-se ao longo do arroio Quarai-chico e, próximo à sua foz, onde é mais preservada, apresenta uma maior variedade florística (espécies arbóreas) do que a formação de SE (GALVANı $\&$ BAPTISTA 2004)

No entorno do parque (EN) foram delimitados como locais de estudo, duas áreas com fitofisionomia semel hante às do PEE. Foi escolhida uma área com formação de SE, localizada em uma propriedade particular, à cerca de $15 \mathrm{~km}$ do parque e uma área de MC, na confluência dos rios Uruguai e Quarai. Neste local a Mata Ciliar apresenta maior heterogeneidade ambiental do que às $M$ atas Ciliares do PEE e árvores com maior porte, que variam entre 20 e $30 \mathrm{~m}$ de altura, além de um estrato de arvoretas (PASTORE et al. 1986).

\section{Amostragem}

Foram real izadas quatro expedições a campo nos períodos de 21 a 25 de março, 15 a 18 de maio, 17 a 20 de setembro de 2003 e 10 a 13 de janeiro de 2004. Foram amostradas áreas com vegetação deSE eMC no parquee no entorno. O inventariamento foi realizado entre 9:00 e 18:00 h predominantemente através de observação direta (busca visual), segundo metodologia adap- tada a partir de PolLARD (1977). Cada área foi percorrida durante três horas por ocasião de amostragem e o esforço amostral foi padronizado em horas de trabalho multiplicadas pelo número de amostradores com rede entomológica (horas-rede).

Todas as espécies citadas foram no mínimo coletadas em uma das expedições, podendo ou não ter sido coletadas em outras, ou apenas visualizadas, mas em ambos os casos, registradas. Para as borboletas visual izadas era anotada a espécie, hora e o local onde foi encontrada. As borboletas coletadas foram acondicionadas em envelopes entomológicos e conduzidas ao laboratório para montagem, sendo dois indivíduos de cada espécie depositados como testemunho na coleção de referência do Laboratório de Ecologia de Insetos, Departamento de Zoologia, Universidade Federal do Rio Grande do Sul (UFRGS). A identificação das espécies foi realizada através de bibliografia especializada (D'ABRERA 1981, 1984, 1987a, b, 1988, 1994, Brown JR. 1992, TYLER et al. 1994, CANALS 2000, 2003) e, conforme o caso, consulta a especialistas. A classificação utilizada para elaboração da listagem de espécies seguiu BRown JR. (1992) e FreitAs \& BRown JR. (2004). A nomenclatura foi atualizada segundo LAMAS (2004). As espécies, dentro das subfamílias, encontram-se listadas em ordem alfabética.

\section{Análise dos dados}

Para a análise dos dados obtidos foram feitas consultas comparativas a inventários de borboletas real izados no Rio Grande do Sul e a uma revisão de gênero. Foram pesquisados os trabalhos de Mabilde (1896), Biezanko \& Freitas (1938), Biezanko \& Seta (1939), Biezanko (1958, 1959a, b, c, 1960a, b, c, d, 1963), BiezANKo \& LinK (1972), BiezANKo \& MielKE (1973), LinK et al. (1977), BiezANKo et al. (1978), Mielke (1980a, b), Ruszczyk (1986), Robiins (1991), Teston \& CoRseuil (1998, 1999, 2000a, b, 2001, 2002), DI Mare et al. (2003), KRüger \& SILva (2003), Iserhard \& RomanowsKI (2004) e QuADros et al. (2004).

A suficiência amostral foi analisada a partir do número de novas espécies coletadas para a região em relação ao número cumulativo de ocasiões amostrais. A riqueza esperada foi estimada através dos índices Chao 1, Jack-knife, Bootstrap e Michaelis-Menten, calculados com o programa EstimateS (Colwell 2004).

Examinou-se ainda a heterogeneidade da distribuição de freqüência de espécies ocorrendo em apenas uma formação vegetal por local de amostragem, espécies estas aqui doravante denominadas "excl".

\section{RESULTADOS E DISCUSSÃO}

Com um total de 300 horas-rede de amostragem, foram registrados 1555 indivíduos de borboletas para o PEE e 961 para o entorno, distribuídos em 97 espécies, pertencentes a cinco famílias e 19 subfamílias (Tab. I).

O número cumulativo de espécies por ocasião de amostragem manteve-se crescente ao longo do estudo (Fig. 1), refletindo a impossibilidade de registrar toda a fauna com um 


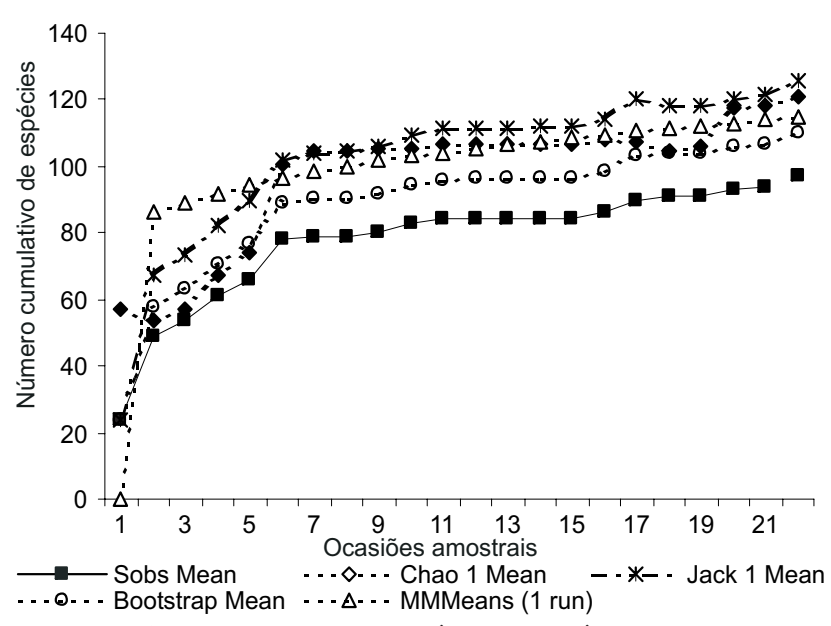

Figura 1. Curva de acumulação (Sobs Mean) e estimadores de riqueza de espécies para borboletas registradas, entre março de 2003 e janeiro de 2004, no Parque Estadual do Espinilho e entorno. Barra do Quarai, Rio Grande do Sul. (Chao 1 Mean) Chao 1, (Jack 1 Mean) Jack-knife, (Bootstrap Mean) Bootstrap e (M M Means 1) Michaelis-Menten.

número restrito de horas de amostragem (BRown JR. \& FreITAS 2000); entretanto, a partir da sétima ocasião amostral, a inclinação da curva torna-se mais suave. A extensão temporal de um inventário tende a aumentar o número total de espécies registradas, além de aumentar a probabilidade de detecção de espécies com tamanhos populacionais baixos (SUMmERVILLE et al. 2001); por outro lado, a proporção de espécies "turistas" tende a crescer na amostra (GASTON 1996) e geralmente é difícil distinguir entre as duas situações. M esmo assim, os estimadores para a riqueza esperada indicam que entre 77 e $88 \%$ das espécies presentes na área de amostragem durante o período de estudo devem ter sido registradas.

Foram registradas 73 espécies de borboletas para o PEE e 83 para EN. A maioria destas pertence à família Nymphalidae (PEE $=37 \%, E=40 \%$ ), seguida por Hesperiidae (PEE $=27 \%, E=$ $23 \%)$, Lycaenidae (PEE $=18 \%, E=16 \%$ ), Pieridae $(\mathrm{PEE}=15 \%, \mathrm{E}$ $=16 \%$ ) e Papilionidae ( $\mathrm{PEE}=3 \%, \mathrm{E}=5 \%$ ). A freqüência relativa da riqueza de espécies por família de borboletas observada no presente estudo, sendo Nymphalidae a família mais rica, seguida por Hesperiidae e Lycaenidae, é similar aquela observada por ISERHARD \& RoMANOWSKI (2004), na região do Vale do Rio Maquiné, nordeste do Rio Grande do Sul. Estes percentuais, entretanto, diferem daquel es registrados para outras regiões do Brasil. BRown JR. \& Freitas (1999) citam para o país Lycaenidae, Hesperiidae e Nymphalidae, nesta ordem, como as mais ricas em espécies. Dados de Mielke \& Casagrande (1997) para o Morro do Diabo (Teodoro Sampaio, São Paulo) e Brown JR. \& Freitas (1999) para o Estado de São Paulo, apontam Hesperiidae como a mais representativa, havendo uma variação nas proporções de riqueza de Nymphalidae e Lycaenidae entre os dois trabalhos. Devido a es- tas diferenças, Mielke \& CASAgRAnde (1997) ressaltam a necessidade de se ampliar este tipo de estudo no sul do país.

É importante salientar que, enquanto no presente inventariamento houve um esforço amostral de 300 horas-rede, Brown JR. \& Freitas (1999) e Mielke \& CASAgrande (1997) apresentam dados que, para certos locais, derivam de vários anos de amostragem. Além disto, segundo Brown JR. \& Freitas (2000), pelas isolinhas de riqueza, o Rio Grande do Sul seria menos rico em espécies do que as áreas onde realizaram a maioria de seus trabalhos. Mesmo assim, destacam-se as diferentes proporções de Lycaenidae e Nymphalidae encontradas, tanto no parque como em seu entorno, em relação aquelas registradas para outras regiões do país.

Teston \& Corseuil (1998, 2000b) listaram 42 espécies de Pieridae e 22 de Papilionidae para o Rio Grande do Sul. Nas regiões de Pelotas, Rio Grande, Missões e Santa Maria foi registrada maior riqueza de espécies de Pieridae do que Papilionidae (Biezanko \& Freitas 1938, Biezanko \& Seta. 1939, Biezanko 1958, 1959a, b, c, LıNK et al. 1977, KRÜGER \& SI LVA 2003). Os resultados aqui obtidos, tanto para PEE como para EN, estão de acordo com esta tendência.

Dez das espécies relacionadas no presente estudo constituem novos registros para o Rio Grande do Sul (Tab. II). Destes novos registros, 70\% pertencem à família Lycaenidae, corroborando a constatação de ISERHARD \& ROMANOWSKI (2004) quanto à importância e a contribuição que os licenídeos podem trazer aos inventariamentos sobre a fauna de borboletas no Estado. Licenídeos apresentam espécies geral mente de tamanho pequeno, difíceis de amostrar e de identificar, sendo esta, provavelmente, uma razão para sua pequena proporção nos registros existentes para o Rio Grande do Sul.

A espécie mais abundante no estudo, com 302 indivíduos registrados, foi Junonia evarete (Cramer, 1779) (Nymphalidae), sendo esta também a espécie mais abundante nas áreas de entorno ( $N=123)$. No PEE a espéciemais abundantefoi Hermeuptychia hermes (Fabricius, 1775) (Nymphalidae) com 216 indivíduos registrados. Ambas podem ser consideradas espécies comuns, generalistas, com ampla distribuição e associadas à ambientes perturbados (DeVRIEs 1987). Adultos de J. evarete visitam grande variedade de flores, inclusive minúsculas ervas de pastiçais ou pradarias ribeirinhas (KLIMAITIS 2000). Hermeuptychia hermes ocorre no extrato herbáceo de campos e ambientes abertos, tendo como hospedei ras diversas espécies de Gramineae (BRown JR. 1992). Os resultados obtidos parecem refletir a dominância da formação vegetal de Savana Estépica Parque na região, com seu vasto tapete de gramíneas e abundância de plantas herbáceas.

A riqueza de espécies encontrada na M ata Ciliar que ocorre dentro do parque foi menor do que aquela encontrada na Mata Ciliar que margeia os rios Uruguai e Quarai. As Matas Ciliares, predominantes no entorno do parque, são de maior porte e apresentam uma flora mais diversa, com mais recursos alimentares, tanto para imaturos quanto para adultos de bor- 
Tabela I. Espécies de borboletas registradas entre março de 2003 e janeiro de 2004 no Parque Estadual do Espinilho e entorno em Barra do Quarai, Rio Grande do Sul. (SE) Savana Estépica, (MC) Mata Ciliar, (S) número de espécies e (*) espécies que ocorreram em apenas uma formação vegetal por local de amostragem (excl).

\begin{tabular}{|c|c|c|c|c|}
\hline \multirow{2}{*}{ Táxon } & \multicolumn{2}{|c|}{ Parque Estadual do Espinilho } & \multicolumn{2}{|c|}{ Entorno } \\
\hline & SE & MC & SE & MC \\
\hline \multicolumn{5}{|l|}{ Nymphalidae $(\mathrm{S}=36)$} \\
\hline \multicolumn{5}{|l|}{ Nymphalinae $(S=9)$} \\
\hline Anartia amathea roeselia (Eschscholtz, 1821) & $x$ & $x$ & $x$ & $x$ \\
\hline Anartia jatrophae (Linnaeus, 1763) & $x$ & $x$ & $x$ & $x$ \\
\hline Anthanassa frisia hermas (Hewitson, 1864) & $x$ & $x$ & & $x$ \\
\hline Eresia lansdorfi (Godart, 1819) * & & & & $x$ \\
\hline Junonia evarete (Cramer, 1779) & $x$ & $x$ & $x$ & $x$ \\
\hline Ortilia ithra (W. F. Kirby, 1900) & $x$ & $x$ & $x$ & $x$ \\
\hline Siproeta stelenes meridionalis (Fruhstorfer, 1909) * & & & & $x$ \\
\hline Tegosa claudina (Eschscholtz, 1821) & & $x$ & $x$ & $x$ \\
\hline Vanessa braziliensis (Moore, 1883) & $x$ & $x$ & $x$ & $x$ \\
\hline \multicolumn{5}{|l|}{ Biblidinae $(S=5)$} \\
\hline Biblis hyperia (Cramer, 1779) & $x$ & $x$ & $x$ & $x$ \\
\hline Dynamine myrrhina (Doubleday, 1849) * & $x$ & & & \\
\hline Eunica eburnea Fruhstorfer, 1907 & & $x$ & & $x$ \\
\hline Eunica maja (Fabricius, 1775) * & & $x$ & & \\
\hline Pyrrhogyra neaerea arge Gosse, 1880 & & $x$ & & $x$ \\
\hline \multicolumn{5}{|l|}{ Satyrinae $(S=5)$} \\
\hline Hermeuptychia hermes (Fabricius, 1775) & $x$ & $x$ & $x$ & $x$ \\
\hline Pampasatyrus periphas (Godart, [1824]) & $x$ & & $x$ & \\
\hline Paryphthimoides phronius (Godart, [1824]) & $x$ & $x$ & $x$ & $x$ \\
\hline Paryphthimoides poltys (Prittwitz, 1865) & $x$ & $x$ & $x$ & \\
\hline Yphthimoides celmis (Godart, [1824]) & $x$ & $x$ & $x$ & $x$ \\
\hline \multicolumn{5}{|l|}{ Heliconiinae $(S=6)$} \\
\hline Agraulis vanillae maculosa (Stichel, [1908]) & $x$ & $x$ & $x$ & $x$ \\
\hline Euptoieta claudia (Cramer, 1775) & $x$ & & $x$ & \\
\hline Dione juno juno (Cramer, 1779) * & & & & $x$ \\
\hline Dryadula phaetusa (Linnaeus, 1758) & & $x$ & & $x$ \\
\hline Dryas iulia alcionea (Cramer, 1779) & $x$ & $x$ & $x$ & $x$ \\
\hline Heliconius erato phyllis (Fabricius, 1775) & & $x$ & & $x$ \\
\hline \multicolumn{5}{|l|}{ Limenitidinae $(\mathrm{S}=1)$} \\
\hline Adelpha thessalia indefecta Fruhstorfer, 1913 & & $x$ & & $x$ \\
\hline \multicolumn{5}{|l|}{ Ithomiinae $(S=3)$} \\
\hline Episcada hymenaea (Prittwitz, 1865) * & & & & $x$ \\
\hline Pseudoscada erruca (Hewitson, 1855) * & & & & $x$ \\
\hline Pteronymia sylvo (Geyer, 1832)* & & & & $x$ \\
\hline \multicolumn{5}{|l|}{ Danainae $(S=2)$} \\
\hline Danaus erippus (Cramer, 1775) & $x$ & $x$ & $x$ & $x$ \\
\hline Danaus gilippus gilippus (Cramer, 1775) & $x$ & $x$ & $x$ & $x$ \\
\hline \multicolumn{5}{|l|}{ Charaxinae $(S=2)$} \\
\hline Memphis moruus stheno (Prittwitz, 1865) * & & & & $x$ \\
\hline \multirow[t]{2}{*}{ Zaretis itys itylus (Westwood, 1850) * } & & & & $x$ \\
\hline & & & & $\mathrm{Co}$ \\
\hline
\end{tabular}


Tabela I. Continuação.

\begin{tabular}{|c|c|c|c|c|}
\hline \multirow{2}{*}{ Táxon } & \multicolumn{2}{|c|}{ Parque Estadual do Espinilho } & \multicolumn{2}{|c|}{ Entorno } \\
\hline & SE & MC & SE & MC \\
\hline \multicolumn{5}{|l|}{ Apaturinae $(S=2)$} \\
\hline Doxocopa kallina (Staudinger, 1886) & $x$ & & $x$ & $x$ \\
\hline Doxocopa laurentia (Godart, [1824]) * & & & & $x$ \\
\hline \multicolumn{5}{|l|}{ Libytheinae (S = 1) } \\
\hline Libytheana carinenta (Cramer, 1777) & $x$ & & $x$ & $x$ \\
\hline \multicolumn{5}{|l|}{ Pieridae (S = 14) } \\
\hline \multicolumn{5}{|l|}{ Coliadinae ( $\mathrm{S}=13$ ) } \\
\hline Aphrissa statira (Cramer, 1777) * & & $x$ & & \\
\hline Colias lesbia lesbia (Fabricius, 1775) & $x$ & $x$ & $x$ & \\
\hline Eurema albula (Cramer, 1775) & & $x$ & & $x$ \\
\hline Eurema deva (Doubleday, 1847) & $x$ & $x$ & & $x$ \\
\hline Eurema elathea (Cramer, 1777) & $x$ & & $x$ & $x$ \\
\hline Eurema phiale (Cramer, 1775) * & & & & $x$ \\
\hline Phoebis argante (Fabricius, 1775) & & $x$ & & $x$ \\
\hline Phoebis neocypris (Hübner, [1823]) & & $x$ & $x$ & $x$ \\
\hline Phoebis philea (Linneaus, 1763) & & $x$ & $x$ & $x$ \\
\hline Phoebis sennae (Linnaeus, 1758) * & & & & $x$ \\
\hline Pyrisitia leuce leuce (Boisduval, 1836) & $x$ & $x$ & $x$ & $x$ \\
\hline Pyrisitia nise tenella (Boisduval, 1836) & & $x$ & & $x$ \\
\hline Rhabdodryas trite banksi (Breyer, 1939) * & & & & $x$ \\
\hline \multicolumn{5}{|l|}{ Dismorphiinae $(\mathrm{S}=1)$} \\
\hline Enantia lina psamathe (Fabricius, 1793) & & $x$ & & $x$ \\
\hline \multicolumn{5}{|l|}{ Papilionidae (S =4) } \\
\hline \multicolumn{5}{|l|}{ Papilioninae $(S=4)$} \\
\hline Battus polydamas polydamas (Linnaeus, 1758) & $x$ & $x$ & & $x$ \\
\hline Heraclides anchisiades capys (Hübner, [1809]) * & & & & $x$ \\
\hline Heraclides astyalus astyalus (Godart, 1819) * & & & $x$ & \\
\hline Heraclides thoas brasiliensis (Rothschild \& Jordan, 1906) & $\mathrm{x}$ & $x$ & $x$ & $x$ \\
\hline \multicolumn{5}{|l|}{ Lycaenidae ( $\mathrm{S}=18$ ) } \\
\hline \multicolumn{5}{|l|}{ Theclinae $(\mathrm{S}=8)$} \\
\hline Calycopis caulonia (Hewitson, 1877) & $\mathrm{x}$ & & $x$ & \\
\hline M inistrymon cruenta (Gosse, 1880) & $x$ & & $x$ & \\
\hline Nicolaea torris (H. H. Druce, 1907) * & & & & $x$ \\
\hline Panthiades hebraeus (Hewitson, 1867) * & & & $x$ & \\
\hline Pseudolycaena marsyas (Linnaeus, 1758) & $x$ & $x$ & $x$ & $x$ \\
\hline Rekoa marius (Lucas, 1857) * & & & & $x$ \\
\hline Strymon cestri (Reakirt, [1867]) * & $x$ & & & \\
\hline Strymon eurytulus (Hübner, [1819]) * & $x$ & & & \\
\hline \multicolumn{5}{|l|}{ Riodininae $(\mathrm{S}=9$ ) } \\
\hline Aricoris signata (Stichel, 1910) & & $x$ & $x$ & \\
\hline Aricoris gauchoana (Stichel, 1910) & & & $x$ & $x$ \\
\hline Aricoris indistincta (Lathy, 1932) * & & & & $x$ \\
\hline Emesis russula Stichel, $1910 *$ & & $x$ & & \\
\hline Euselasia hygenius occulta Stichel, 1919 & & $x$ & & $\mathrm{x}$ \\
\hline
\end{tabular}


Tabela I. Continuação.

\begin{tabular}{|c|c|c|c|c|c|c|c|}
\hline \multirow{2}{*}{ Táxon } & \multicolumn{3}{|c|}{ Parque Estadual do Espinilho } & \multicolumn{4}{|c|}{ Entorno } \\
\hline & SE & & MC & & SE & & MC \\
\hline Euselasia modesta (H. W. Bates, 1868) & & & $x$ & & & & $x$ \\
\hline M elanis aegates (Hewitson, 1874) & & & $x$ & & & & $x$ \\
\hline Riodina lysippoides Berg, 1882 & $x$ & & $x$ & & $x$ & & $x$ \\
\hline Zabuella tenellus (Burmeister, 1878) * & $\mathrm{x}$ & & & & & & \\
\hline \multicolumn{8}{|l|}{ Polyommatinae $(S=1)$} \\
\hline Leptotes cassius (Cramer,1775) * & $x$ & & & & & & \\
\hline \multicolumn{8}{|l|}{ Hesperiidae $(\mathrm{S}=25)$} \\
\hline \multicolumn{8}{|l|}{ Hesperiinae $(S=7)$} \\
\hline Cymaenes gisca Evans, 1955 * & & & & & & & $x$ \\
\hline Hylephila phyleus (Drury, 1773) & $x$ & $x$ & & $x$ & & $x$ & \\
\hline Lerodea eufala eufala (W. H. Edwards, 1869) * & $x$ & & & & & & \\
\hline Nyctelius nyctelius nyctelius (Latreille, [1824]) & $x$ & $x$ & & & & $x$ & \\
\hline Panoquina ocola ocola (W. H. Edwards, 1863) & $x$ & $x$ & & & & $x$ & \\
\hline Polites vibex catilina (Plötz, 1886) & $x$ & $x$ & & & & & \\
\hline Pompeius amblyspila (Mabille, 1898) * & $x$ & & & & & & \\
\hline \multicolumn{8}{|l|}{ Pyrginae $(S=16)$} \\
\hline Chioides catillus catillus (Cramer, 1779) & & $x$ & & & & $x$ & \\
\hline Erynnis (Erynnides) funeralis (Scudder \& Burgess, 1870) * & $x$ & & & & & & \\
\hline Gesta austerus (Schaus, 1902) * & $x$ & & & & & & \\
\hline Gorgythion beggina escalophoides Evans, 1953 & $x$ & & & & & $x$ & \\
\hline Heliopetes omrina (Butler, 1870) & $x$ & $x$ & & $x$ & & $x$ & \\
\hline Mylon maimon (Fabricius, 1775) * & & & & & & $x$ & \\
\hline Nisoniades bipuncta (Schaus, 1902) * & $x$ & & & & & & \\
\hline Pyrgus orcus (Stoll, 1780) & $x$ & $x$ & & $x$ & & $x$ & \\
\hline Pyrgus orcynoides (Giacomelli, 1928) & $x$ & & & $x$ & & $x$ & \\
\hline Urbanus dorantes dorantes (Stoll, 1790) & $x$ & $x$ & & $x$ & & & \\
\hline Urbanus procne (Plötz, 1880) & $x$ & $x$ & & & & $x$ & \\
\hline Urbanus proteus proteus (Linnaeus, 1758) & & & & $x$ & & $x$ & \\
\hline Urbanus simplicius (Stoll, 1790) & $x$ & $x$ & & $x$ & & $x$ & \\
\hline Urbanus teleus (Hübner, 1821) & $x$ & $x$ & & $x$ & & $x$ & \\
\hline Viola minor (Hayward, 1933) * & & & & $x$ & & & \\
\hline Xenophanes tryxus (Stoll, 1780) & $x$ & $x$ & & & & $x$ & \\
\hline \multicolumn{8}{|l|}{ Pyrrhopyginae ( $\mathrm{S}=2)$} \\
\hline Mysoria barcastus barta Evans, 1951 * & & & & & & $x$ & \\
\hline Pyrrhopyge aziza subnubilus Hayward, 1935 & & $\mathrm{x}$ & & & & $x$ & \\
\hline Número de espécies por formação vegetal em cada localidade & 52 & 54 & & 42 & & 72 & \\
\hline
\end{tabular}

boletas. Sugere-se ser esta a causa da diferença observada na riqueza de borboletas entre ambos locais.

Trinta e cinco espécies foram coletadas em somente um tipo de formação vegetal e apenas no parque ou em seu entorno (excl): três em MC no PEE, 19 em MC no E, 10 em SE no PEE e 3 em SE no E (Tab. I). A maioria das espécies excl ocorreu na
Mata Ciliar do entorno do parque, entretanto, a diferença mais marcante em relação ao esperado diz respeito àquelas espécies que ocorreram somente na SE do PEE $\left(\chi^{2}=13,96\right.$; $\mathrm{gl}=1 ; \mathrm{p}<$ 0,001). Com exceção de Dynamine myrrhina (Doubleday, 1849) (Nymphalidae), que prefere o ambiente das florestas (BRown JR. 1992), a maioria das espécies excl encontradas em SE do PEE 
Tabela II. Novos registros de espécies de borboletas para o Rio Grande do Sul. Espécies coletadas entre março de 2003 e janeiro de 2004 no Parque Estadual do Espinilho e entorno no município de Barra do Quarai, Rio Grande do Sul. É fornecida a data da primeira coleta para cada espécie.

\begin{tabular}{|c|c|c|}
\hline Família/subfamília & Espécie & $\begin{array}{c}\text { Data de } \\
\text { coleta }\end{array}$ \\
\hline \multicolumn{3}{|l|}{ Nymphalidae } \\
\hline Nymphalinae & Anthanassa frisia hermas & 22.III.2003 \\
\hline Biblidinae & Pyrrhogyra neaerea arge & 22.III.2003 \\
\hline \multicolumn{3}{|l|}{ Lycaenidae } \\
\hline \multirow[t]{4}{*}{ Theclinae } & Ministrymon cruenta & 24.III.2003 \\
\hline & Nicolaea torris & 11.1.2004 \\
\hline & Rekoa marius & 24.III.2003 \\
\hline & Strymon cestri & 12.I.2004 \\
\hline \multirow[t]{3}{*}{ Riodininae } & Aricoris indistincta & 11.1 .2004 \\
\hline & Euselasia modesta & 22.III.2003 \\
\hline & Melanis aegates & 22.III.2003 \\
\hline \multicolumn{3}{|l|}{ Hesperiidae } \\
\hline Pyrrhopyginae & Pyrrhopyge aziza subnubilus & 22.III.2003 \\
\hline
\end{tabular}

são general istas e associadas a áreas abertas e de campo (Brown JR. 1992, CANALS 2000, 2003). As lagartas de Leptotes cassius (Cramer,1775) (Lycaenidae) e Erynnis (Erynnides) funeralis (Scudder \& Burgess, 1870) (Hesperiidae) se alimentam de várias espécies de Fabaceae (CANALS 2003), enquanto que Lerodea eufala eufala (W. H. Edwards, 1869) (Hesperiidae) tem como planta hospedeira espécies do gênero Cyperus (Cyperaceae) (BıEZANKo et al. 1957). A vegetação campestre de SE do PEE é rica em espécies, principalmente das famílias Fabaceae e Cyperaceae, quando comparada com outras regiões da fronteira oeste do Rio Grande do Sul (GALVANI \& BAPTISTA 2004). Sugerese portanto que, a pesar de seu tamanho reduzido, a formação de SE que se encontra dentro dos limites do parque, por apresentar uma vegetação herbácea bastante diversificada, exerça uma importante contribuição para a composição local da fauna de borboletas. Chama atenção a presença de Strymon cestri (Reakirt, [1867]) (Lycaenidae) entre as espécies excl de SE do PEE, citada pela primeira vez para o Rio Grande do Sul através do presente trabalho. Também merece atenção Zabuella tenellus (Burmeister, 1878) (Lycaenidae) considerada espécie rara no Estado (BıeZAnKo et al. 1978, KRüger \& Silva 2003).

Inventariamentos a curto prazo podem revelar características importantes de comunidades locais, tais como a utilização de recursos e a preferência de habitat, além de contribuir para o conhecimento da fauna regional. O presente estudo foi desenvolvido em tempo exíguo. Apesar disto, pode-se evidenciar a importância da Savana Estépica Parque para a composição da lepidopterofauna da região. Quando uma determinada área passa a integrar uma UC, os estudos ali realizados serão diretamente aplicáveis aos usos da mesma de modo a contribuir para que sejam atingidas as finalidades que motivaram o seu estabelecimento como área silvestre especialmente protegida (Oliveira 1988). Além disto, quanto mais se saiba sobre uma área protegida, melhor poderá ser o planejamento da proteção e uso da mesma (Dourojeannı \& PÁdua 2001). Assim sendo, espera-se que o presente estudo possa ser útil a futuras ações de manejo do Parque Estadual do Espinilho e do seu entorno, além de contribuir para ampliar o conhecimento sobre a lepidopterofauna do Rio Grande do Sul.

\section{AGRADECIMENTOS}

Aos colegas do Laboratório de Ecologia de Insetos (UFRGS) e a Melissa Oliveira Teixeira, Lucas Kaminski, Cristiano Agra Iserhard e Milton Mendonça Jr. Aos doutores André V.L. Freitas (UNICAMP), Olaf H.H. Mielke (UFPR), Robert Robbins (Smithsonian Institution) e Curtis Callaghan pelas valiosas identificações das borboletas. Ao CNPq pelo financiamento (Processo 478787/2001-4).

\section{REFERÊNCIAS BIBLIOGRÁFICAS}

Biezanko, C.M. 1958. Pieridae da Zona Sueste do Rio Grande do Sul. Arquivos de Entomologia, Série A, Pelotas: 1-15.

BiezAnKo, C.M. 1959a. Papilionidae da Zona Sueste do Rio Grande do Sul. Arquivos de Entomologia, Série A, Pelotas: 117.

Bıezanko, C.M. 1959b. Papilionidae da Zona Missioneira do Rio Grande do Sul. Arquivos de Entomologia, Série B, Pelotas: 1-12.

BieZAnKo, C.M. 1959c. Pieridae da Zona Missioneira do Rio Grande do Sul. Arquivos de Entomologia, Série B, Pelotas: 112

BiezAnKo, C.M. 1960a. Danaidae et Ithomidae da Zona Sueste do Rio Grande do Sul. Arquivos de Entomologia, Série A, Pelotas: 1-6.

Biezanko, C.M. 1960b. Danaidae et Ithomidae da Zona Missioneira do Rio Grande do Sul. Arquivos de Entomologia, Série B, Pelotas: 1-6.

BiezAnKo, C.M. 1960c. Satyridae, Morphidae et Brassolidae da Zona Sueste do Rio Grande do Sul. Arquivos de Entomologia, Série A, Pelotas: 1-13.

BiezAnKo, C.M. 1960d. Satyridae, Morphidae et Brassolidae da Zona Missioneira do Rio Grande do Sul. Arquivos de Entomologia, Série B, Pelotas: 1-10.

Bıezanko, C.M. 1963. Hesperiidae da Zona Sueste do Rio Grande do Sul. Arquivos de Entomologia, Série A, Pelotas: 125.

Biezanko, C.M.; A. Ruffinelli \& C.S. Carbonell. 1957. Lepidoptera del Uruguay. Lista anotada de especies. Revista de la Facultad de Agronomia, Montevideo, (46): 1-115.

BiezAnko, C.M. \& D. Link. 1972. Nomes populares dos lepidópteros no Rio Grande do Sul. (Segundo Catálogo). Nomes 
populares - nomes científicos atualizados. Boletim Técnico Defesa Fitosanitária da Universidade Federal de Santa Maria, Santa Maria, 4: 1-15.

Biezanko, C.M. \& F.D. SetA. 1939. Catálogo dos insetos encontrados em Rio Grande e seus arredores. Fasc. 1. Lepidopteros. Pelotas, "A Universal” - Echenique, 15p.

Biezanko, C.M. \& O.H.H. MıelKe. 1973. Contribuição ao estudo faunístico dos Hesperiidae americanos. IV. Espécies do Rio Grande do Sul, Brasil, com notas taxonômicas e descrições de espécies novas (Lepidoptera). Acta Biológica Paranaense, Curitiba, 2 (1-4): 51-102.

Biezanko, C.M.; O.H.H. Mielke \& A. Wedderhoof. 1978. Contribuição ao estudo faunístico dos Riodinidae do Rio Grande do Sul, Brasil (Lepidoptera). Acta Biológica Paranaense, Curitiba, 7 (1): 7-22.

Biezanko, C.M. \& R.G. Freitas. 1938. Catálogo dos insetos encontrados na cidade de Pelotas e seus arredores. Fasc. 1. Lepidopteros. Pelotas, Escola de Agronomia Eliseu Maciel, 32p.

Brito, M.C.W.; L.P. Vianna; C.M.A. Azevedo; F.P. Fonseca; R.R. Mendonça \& D.M.D. Carvalho. 1999. Unidades de Conservação, p. 3-44. In: M.C.W. BRITo \& C.A. Joly (Eds). Biodiversidade do Estado de São Paulo, Brasil: síntese do conhecimento ao final do século XX. São Paulo, FAPESP, vol. 7, 150p.

Brown JR, K.S. 1992. Borboletas da Serra do Japi: diversidade, hábitats, recursos alimentares e variação temporal, p. 142187. In: L.P. Morellato (Ed.). História Natural da Serra do Japi: ecologia e preservação de uma área florestal no sudeste do Brasil. Campinas, Editora da UNICAMP/FAPESP, $321 p$.

BRoWn JR, K.S. 2001. Insetos indicadores da história, composição, diversidade e integridade de Matas Ciliares, p. 223-232. In: R.R. Rodrigues \& H.F. LeitÃo-Filho (Eds.). Matas Ciliares: conservação e recuperação. São Paulo, EDUSP, $2^{\text {a }}$ ed., 320p.

Brown JR, K.S. \& A.V.L. FreitAS. 1999. Lepidoptera, p. 225-245.

In: C.R.F. Brandão \& E.M. Cancello (Eds). Biodiversidade do Estado de São Paulo, Brasil. São Paulo, FAPESP, $X V I+279 p$.

Brown JR., K.S. \& A.V.L. FreitAs. 2000. Atlantic Forest Butterflies: indicator for landscape conservation. Biotropica, Washington, 32 (4b): 934-956.

Bruner, A.G.; R.E. Gullison; R.E. Rice \& G.A.B. Fonseca. 2001. Effectiveness of parks in protecting tropical biodiversity. Science, Washington, 291 (5501): 125-127.

BudKe, J.C.; E.L.H. Giehl; E.A. Athayde, S.M. Eisinger \& R.A. ZÁchiA. 2004. Florística e fitossociologia do componente arbóreo de uma floresta ribeirinha, arroio Passo das Tropas, Santa Maria, RS, Brasil. Acta Botânica Brasilica, São Paulo, 18 (3): 581-589.

Canals, G.R. 2000. Mariposas Bonaerenses. Buenos Aires, L.O.L.A., 347p.

Canals, G.R. 2003. Mariposas de Misiones. Buenos Aires,
L.O.L.A., 492p.

ColwelL, R.K. 2004. EstimateS: Statistical estimation of species richnessand shared speciesfrom samples. Version 7.0. User's Guide and application. Available in the World Wide Web in: http://purl.oclc.org/estimates [Accessed in 14.06.2005].

D'Abrera, B. 1981. Butterflies of the Neotropical Region. Part I. Papilionidae \& Pieridae. Victoria, Hill House, XIV +172p.

D'Abrera, B. 1984. Butterflies of the Neotropical Region. Part II. Danaidae, Ithomidae, Heliconidae \& Morphidae. Victoria, Hill House, XII+210p.

D'Abrera, B. 1987a. Butterflies of the Neotropical Region. Part III. Brassolidae, Acraeidae \& Nymphalidae (partim). Victoria, Hill House, IX $+139 p$.

D'Abrera, B. 1987b. Butterflies of the Neotropical Region. Part IV. Nymphalidae (partim). Victoria, Hill House, XV+150p.

D'Abrera, B. 1988. Butterflies of the Neotropical Region. Part V. Nymphalidae (conc.) \& Satyridae. Victoria, Hill House, IX+197p.

D'Abrera, B. 1994. Butterflies of the Neotropical Region. Part VI. Riodinidae. Victoria, Hill House, IX $+216 p$.

DeVRIES, P.J. 1987. The butterflies of Costa Rica and their natural history. Vol. I. Papilionidae, Pieridae and Nymphalidae. New Jersey, Princeton University, 327p.

Dı Mare, R.A.; J.A. Teston \& E. Corseuil. 2003. Espécies de Adelpha Hübner, [1819] (Lepidoptera, Nymphalidae, Limenitidinae) ocorrentes no Rio Grande do Sul, Brasil. Revista Brasileira de Entomologia, São Paulo, 47 (1): 75-79.

Dourojeanni, M.J. \& M.T.J. PÁduA. 2001. Biodiversidade: a hora decisiva. Curitiba, Editora da UFPR, 308p.

FreitAs, A.V.L. \& K.S. Brown JR. 2004. Phylogeny of the Nymphalidae (Lepidoptera). Systematic Biology, Washington, 53 (3): 1-25.

Galvani, F.R. \& L.R.M. BaptistA. 2004. Flora do Parque Estadual do Espinilho, Barra do Quarai, RS. Revista da Faculdade de Zootecnia, Veterinária e Agronomia, Uruguaiana, 10: 148168.

GASTON, K.J. 1996. Biodiversity. A biology of numbers and difference. Oxford, Blackwell Science, 396p.

ISERHARD, C.A. \& RomANowskI, H.P. 2004. Lista de espécies de borboletas (Lepidoptera, Papilionoidea e Hesperioidea) da região do vale do rio Maquiné, Rio Grande do Sul, Brasil. Revista Brasileira de Zoologia, Curitiba, 21 (3): 649-662.

KLIMAITIS, J.F. 2000. Cien mariposas Argentinas. Buenos Aires, Albatros, $128 p$.

KrÜGer, C.P. \& E.J.E. Silva. 2003. Papilionoidea (Lepidoptera) de Pelotas e seus arredores, Rio Grande do Sul, Brasil. Entomologia y Vectores, Rio de Janeiro, 10 (1): 31-45

LAMAS, G. (Ed.). 2004. Atlas of Neotropical Lepidoptera. Checklist: Part 4A. Hesperioidea \& Papilionoidea. Gainesville, Scientific Publishers, XXXVI+439p.

Link, D.; C.M. Biezanko; M.F. Tarragó \& S. Carvalho. 1977. Lepidoptera de Santa Maria e arredores. I. Papilonidae e Pieridae. Revista do Centro de Ciências Rurais, Santa Ma- 
ria, 7 (4): 381-389.

MABILDE, A.P. 1896. Guia practica para os principiantes colleccionadores de insectos, contendo a descripção fiel de perto de 1000 borboletas com 280 figuras lythographadas em tamanho, formas e dezenhos conformeo natural. Estudo sobrea vida de insectos do Rio Grande do Sul e sobre a caça, classificação e conservação de uma collecção mais ou menos regular. Porto Alegre, Gundlach \& Schuldt, 238p.

MARCHIORI, J.N.C.; S.J. LoNGHI \& L. GALVÃo. 1985. Composição florística e estrutura do Parque do Inhanduvaí no Rio Grande do Sul. Revista do Centro de Ciências Rurais, Santa Maria, 15 (4): 319-334.

MielKe, O.H.H. 1980a. Contribuição ao estudo faunístico dos Hesperiidae americanos. V. Nota suplementar - As espécies de Pyrrhopyginae e Pyrginae do Rio Grande do Sul, Brasil (Lepidoptera). Acta Biológica Paranaense, Curitiba, 8-9: 7-17.

MielKe, O.H.H. 1980b. Contribuição ao estudo faunístico dos Hesperiidae americanos. VI. Nota suplementar - As espécies de Hesperiinae do Rio Grande do Sul, Brasil (Lepidoptera). Acta Biológica Paranaense, Curitiba, 8-9: 127-172.

Mielke, O.H.H. \& M.M. Casagrande. 1997. Papilionoidea e Hesperioidea (Lepidoptera) do Parque Estadual do Morro do Diabo, Teodoro Sampaio, Brasil, com notas taxonômicas sobre Hesperiidae. Revista Brasileira de Zoologia, Curitiba, 14 (4): 967-1001.

MorelLato, L.P.C. 1992. Introdução, p. 8-11. In: L.P.C. MoReLLATO (Ed.). História Natural da Serra do Japi: Ecologia de uma Área Florestal no Sudeste do Brasil. Campinas, Editora da UNICAMP/FAPESP, 321p.

MotA, F.S. 1951. Estudos do clima do Estado do Rio Grande do Sul, segundo o sistema de W. Köppen. Revista Brasileira de Geografia, Rio de Janeiro, 13 (2): 275-284.

Oliveira, M.L.A.A. 1988. Vegetação de macrófitas aquáticas das nascentes do rio Gravataí (banhado Grande banhado Chico Lomã), Rio Grande do Sul, Brasil - levantamento preliminar. Iheringia, Série Botânica, Porto Alegre, (38): 67-80.

Pastore, U.; A.L.R.R. Filho; M.B. Teixeira \& A.B.C. Neto. 1986. Vegetação - Projeto RADAMBRASIL. Levantamento de recursos naturais. Rio de Janeiro, IBGE, vol. 33, p. 541-620.

PolLARD, E. 1977. A method for assessing changes in theabundance of butterflies. Biological Conservation, London, 12 (2): 115134

Quadros, F. do C.; A.L. Dorneles \& E. Corseuil. 2004. Ninfalídeos (Lepidoptera, Nymphalidae) ocorrentes no norte da planície costeira do Rio Grande do Sul, Brasil. Biociências, Porto Alegre, 12 (2): 147-164.

Rambo, S.J.B. 1994. A fisionomia do Rio Grande do Sul. São
Leopoldo, Unisinos, 3a ed., 474p.

RoвBINS, R.K. 1991. Evolution, comparative morphology and identification of the Eumaeine butterfly genus Rekoa Kaye (Lycaenidae: Theclinae). Smithsonian Contributions to Zoology, Washington, (498): 1-64.

RuszczYK, A. 1986. Ecologia urbana de borboletas. I. O gradiente de urbanização e a fauna de Porto Alegre, RS. Revista Brasileira de Biologia, Rio de Janeiro, 46 (4): 675-688.

SAntos, E.R.O. 1991. Estudo fisio-geográfico do Parque Estadual do Espinilho, município de Uruguaiana, RS. Boletim Informativo do Instituto Geobiológico La Salle, Canoas, (10): 1-91.

Soulé, M.E. \& J. Terborgh. 1999. The policy and science of regional conservation, p. 1-17. In: M.E. SOulÉ \& J. Terborgh. (Eds). Continental Conservation: scientific foundations of re gional reserve networks. Washington, Island Press, 227p.

Summerville, K.S.; E.H. Metzler \& T.O. CRISt. 2001. Diversity of Lepidoptera in Ohio forests at local and regional scales: how heterogeneous is the fauna? Annals of the Entomological Society of America, Ohio, 94 (4): 583-591.

Teston, J.A. \& E. Corseuil. 1998. Lista documentada dos papilionídeos (Lepidoptera, Papilionoidea) do Rio Grande do Sul, Brasil. Biociências, Porto Alegre, 6 (2): 81-94.

Teston, J.A. \& E. Corseull. 1999. Borboletas (Lepidoptera, Rhopalocera) ocorrentes no Centro de Pesquisas e Conservação da Natureza Pró-Mata. 1. Papilionidae. Biociências, Porto Alegre, 4: 217-228.

Teston, J.A. \& E. Corseuil. 2000a. Borboletas (Lepidoptera, Rhopalocera) ocorrentes no Centro de Pesquisas e Conservação da Natureza Pró-Mata. 2. Pieridae. Biociências, Porto Alegre, 5: 143-155.

Teston, J.A. \& E. Corseuil. 2000b. Lista documentada dos pierídeos (Lepidoptera, Pieridae) do Rio Grande do Sul, Brasil. Biociências, Porto Alegre, 8 (2): 115-132.

Teston, J.A. \& E. Corseuil. 2001. Ninfalídeos (Lepidoptera, Nymphalidae) ocorrentes no Rio Grande do Sul, Brasil. Parte I. Danainae e Ithomiinae. Biociências, Porto Alegre, 9 (1): 51-61.

Teston, J.A. \& E. Corseuil. 2002. Borboletas (Lepidoptera, Rhopalocera) ocorrentes no Centro de Pesquisas e Conservação Pró-Mata. 3. Nymphalidae. Divulgação do Museu de Ciências e Tecnologia da PUCRS, Porto Alegre, (7): 79-125.

TYLER, H.; K.S. BROWN JR. \& K. WILSON. 1994. Swallowtail Butterflies of the Americas. Florida, Scientific Publishers, 376p.

Veloso, H.P.; A.L.R.R. Filho \& J.C.A. Lıma 1991. Classificação da vegetação brasileira adaptada a um sistema universal. Rio de Janeiro, IBGE, 124p.

WILSON, E.O. 1997. Biodiversidade. Rio de Janeiro, Nova Fronteira, 700p.

Recebido em 16.II.2006; aceito em 28.IX.2006. 